\title{
Un estudio exploratorio sobre los errores y las dificultades del alumnado de Bachillerato respecto al concepto de derivada
}

\author{
Antonio González-García ${ }^{* *}$ ), Laura Muñiz-Rodríguez $\left(^{*}\right)$, Luis José Rodríguez-Muñiz (*) \\ (*) Universidad de Oviedo - España (**) Educación Secundaria - Asturias - España
}

\begin{abstract}
RESUMEN
Los errores que comete el alumnado al resolver una tarea matemática suponen una herramienta poderosa para detectar dificultades en el aprendizaje de esta disciplina. Su diagnóstico permite el desarrollo de técnicas de enseñanza que eviten o remedien su cometido. Este artículo presenta los resultados de un estudio exploratorio cuyo objetivo es analizar los errores que comete el alumnado al estudiar el concepto de derivada de una función. Para ello se examinaron los errores cometidos por 27 estudiantes de $1^{\circ}$ de Bachillerato durante la resolución de una serie de ejercicios. Los resultados permiten identificar aquellas categorías en las que los estudiantes cometen mayores errores en el estudio de la derivada. El análisis también revela dificultades causadas por un aprendizaje deficiente de conocimientos previos. Todo ello invita a reflexionar sobre la necesidad de fomentar un análisis lógico y coherente de los conceptos, del lenguaje y de las soluciones, a la hora de resolver problemas matemáticos.
\end{abstract}

Palabras Clave: Bachillerato, Derivada, Dificultades, Errores, Matemáticas.

\section{An exploratory study about the errors and difficulties of high school students regarding the concept of derivative}

\begin{abstract}
The errors that students make when solving a mathematical problem are a powerful tool to detect difficulties in the learning of this discipline. Their diagnosis allows the development of teaching techniques that avoid or rectify their commission. This article presents the results of an exploratory study which aims at analyzing the errors that students make when studying the concept of derivative of a function. To this end, the errors made by 27 high school students during the resolution of a series of exercises are examined. The results allow to identify those categories in which students make a higher number of errors when studying the derivative. The analysis also reveals difficulties caused by poor learning of prior knowledge. The later invites to reflect on the need to promote a logical and coherent analysis of concepts, language and solutions, when solving mathematical problems.
\end{abstract}

Keywords: Derivative, Difficulties, Errors, High school, Mathematics.

\section{Introducción}

El estudio y análisis de los errores que comete el alumnado al resolver tareas matemáticas es una fuente primordial de retroalimentación en el proceso de enseñanza-aprendizaje. De la información que proporcionan estos procesos, el docente y el investigador deben extraer pautas que sirvan para mejorar los procesos del aula y los enfoques educativos que se puedan diseñar.

La enseñanza y el aprendizaje de la derivada, en sus múltiples facetas (geométrica, funcional, algebraica, etc.) ha sido objeto de estudio tanto en la Educación Secundaria como en la Educación Superior. El estudio que se presenta plantea un análisis de los errores cometidos respecto a la derivada, su cálculo e interpretación por alumnado de $1^{\underline{0}}$ de Bachillerato. El trabajo comienza con una descripción del marco teórico de la investigación, explicando las diferentes clasificaciones de errores y sus particularizaciones al ámbito de la derivada, y mostrando la investigación más reciente respecto al aprendizaje de este concepto. A continuación, se describe el desarrollo metodológico, i.e., el objetivo del estudio, los sujetos de investigación, y el procedimiento para la recogida y análisis de datos. Posteriormente, se realiza el análisis de los errores observados y se lleva a cabo una clasificación de estos. Finalmente, se proponen una serie de medidas desarrolladas a partir de los resultados del estudio para solventar las dificultades en el aprendizaje de la derivada en el alumnado de $1^{\circ}$ de Bachillerato. 


\section{Marco teórico}

\section{Análisis de errores}

El error debe ser considerado como la presencia en el alumnado de un esquema cognitivo inadecuado, no solo como una falta de conocimiento o una distracción (Socas, 1997). La detección, estudio y diagnóstico de errores se ha convertido en un aspecto relevante dentro de la investigación en educación matemática. Rico (1998) propone cuatro líneas de investigación respecto a errores en el aprendizaje de las matemáticas: análisis de errores, tratamiento curricular de los errores, formación del profesorado en la detección y corrección de errores, y otros trabajos de carácter técnico (como análisis estadístico de errores).

Según Mulhern (cit. por Engler, Gregorini, Müller, Vrancken, y Hecklein, 2004), las características generales de los errores en matemáticas son:

- Los errores surgen de manera espontánea en clase y son cometidos de forma inconsciente por parte de los estudiantes.

- Los errores son predominantemente sistemáticos, es decir, son el reflejo de una comprensión equivocada, como resultado de fallos en la asimilación de contenidos o conceptos fundamentales en matemáticas.

- La comisión de errores conlleva un desconocimiento de los conceptos y símbolos utilizados, así como una actitud acrítica hacia los resultados obtenidos.

Varios autores han establecido clasificaciones de los errores más comunes, de acuerdo con diferentes estándares (Movshovitz-Hadar, Zaslavsky, y Inbar, 1987; Radatz, 1979, 1980; Socas, 1997). Históricamente se considera que Weiner, en 1922, fue el primero en abordar el estudio de los errores como una herramienta útil para la mejora de aprendizaje (Rico, 1998). Dentro del ámbito de las matemáticas, una de las clasificaciones más utilizadas es la de Radatz $(1979,1980)$, basada en las teorías del procesamiento de la información. En ella se establecen cinco categorías (Rico, 1998):

- Errores debidos a dificultades de lenguaje: la transición del lenguaje coloquial al lenguaje formal de las matemáticas para resolver los problemas, y la dificultad que supone la utilización del lenguaje y la notación matemática, lejanos para el alumnado.

- Errores debidos a dificultades para obtener información espacial: los que se pueden cometer en representaciones icónicas de determinadas situaciones matemáticas, al procesar la información.

- Errores debidos a un aprendizaje deficiente de hechos, destrezas y conceptos previos: los debidos al desconocimiento de procedimientos y conceptos básicos para llevar a cabo satisfactoriamente una determinada tarea matemática.

- Errores debidos a asociaciones incorrectas o a rigidez del pensamiento: los que aparecen cuando procedimientos y conceptos matemáticos que los estudiantes aprenden en un contexto concreto deben ser aplicados en contextos diferentes. Esta categoría se divide a su vez en otras cinco: errores por perseveración, de asociación, de interferencia, de asimilación, y de transferencia negativa a partir de tareas previas.

- Errores debidos a la aplicación de reglas o estrategias irrelevantes: los derivados de procedimientos y destrezas que, aunque correctamente aplicados en determinados contextos, se trasladan incorrectamente al aplicarlos en áreas diferentes.

Esta clasificación es utilizada posteriormente en numerosas investigaciones. Franchi y Hernández de Rincón (2004) la utilizan para proponer una tipología de los errores en el área de la geometría plana. Bocco y Canter (2010) para realizar un análisis de los errores que aparecen en la resolución de problemas en geometría básica, en el que constatan un alto porcentaje de errores debidos a un aprendizaje deficiente de hechos, destrezas, y conceptos previos. En relación con el concepto de derivada, Martínez Jurado y Francisco Solano (2010) estudian las dificultades que tienen los estudiantes de nivel superior para relacionar la derivada en sus diferentes representaciones: algebraica, numérica, razón de cambio y forma gráfica. Concluyen que las principales dificultades según la clasificación de Radatz, fueron la de lenguaje, distinción de datos, y aprendizaje deficiente previo.

Recientemente, Rodríguez-Muñiz y Candás (2017) analizan los errores de un grupo de más de 600 estudiantes al resolver un límite en las pruebas de acceso a la universidad. Se demostró que la gran mayoría de los errores, con el modelo de Radatz, estaban en la categoría de errores por aprendizaje deficiente de hechos, destrezas y conceptos previos.

\section{El aprendizaje de la derivada}

Sánchez-Matamoros, García, y Llinares (2008) presentan un estado del arte sobre la investigación acerca del aprendizaje de la derivada. Uno de los primeros trabajos sobre la derivada se lo debemos a Orton (1983), quien determina tres tipos de errores: estructurales (relacionados con lo conceptual), arbitrarios (se producen sin razón aparente) y de manipulación (manejo incorrecto del lenguaje o de las reglas). Artigue (1995) señala la relativa facilidad para adiestrar al alumnado en los métodos y reglas de derivación, frente a la mayor dificultad para que los conceptos que se ponen en juego sean correctamente comprendidos.

Los trabajos de Azcárate (cit. por Ortega y Sierra, 1998) fueron pioneros en España en el estudio del aprendizaje de la derivada. Azcárate, Casadelvall, Casellas, y Bosch (1996) distinguen tres perfiles cognitivos de los estudiantes ante el concepto de derivada, atendiendo a su interpretación como tasa de variación:

- Perfil primitivo: no se llega a construir el concepto de velocidad o tasa de variación instantánea.

- Perfil de aproximación: se aproxima el concepto de velocidad o tasa de variación instantánea a partir de la velocidad o tasa media entre dos puntos próximos como.

- Perfil límite: se han interiorizado los conceptos de velocidad y tasa de variación instantánea, y se emplean correctamente.

También Azcárate (1990) identificó errores comunes como la confusión entre el valor de la pendiente de la recta tangente en un punto con el de la ordenada en el origen.

Asiala, Cottrill, Dubinsky, y Schwingendorf (1997), utilizando el marco de la teoría APOE (Acción, Proceso, Objeto, Esque$\mathrm{ma}$ ), analizaron cómo se produce la construcción mental cuando se estudian conjuntamente la interpretación gráfica de una función y de su derivada. Sus resultados resaltan la interconexión geométrica-analítica en la comprensión del concepto de derivada. También el trabajo de Clark et al. (1997) se enmarca en esta teoría, identificando tres niveles de análisis para la regla de la cadena. 
Para otros autores, la teoría APOE resulta insuficiente ya que obvia los problemas relacionados con la representación y el significado. Así, Font (2000) subraya los problemas basados en el deficiente aprendizaje de conceptos previos (como función o límite) necesarios para desarrollar la idea de derivada. Font concluye, además, que la idea de tasa de variación se construye mejor cuando se utiliza la tabla como apoyo en la representación de los valores.

También encontramos aproximaciones al aprendizaje de la derivada desde la teoría socioepistemológica. Cantoral y Farfán (2003) subrayan la importancia de alejarse del límite del cociente o de la tangente, para acercarse a la derivada insistiendo en el concepto de variación sucesiva y en la posibilidad de modelar fenómenos de cambio (Montiel, 2005).

Desde el punto de vista de las teorías de la reificación, Zandieth (2000) plantea que, para lograr una adecuada reificación de la idea de derivada, debemos considerar la imagen de un puzle mental en el cual las piezas son las representaciones gráfica, verbal, física y simbólica de la derivada.

Badillo (2003) realiza una descomposición genética del concepto de derivada en el desarrollo de su aprendizaje. También da a conocer la existencia de diferentes significados de la idea de derivada en un punto y de función derivada. En su estudio concluye que entre el profesorado se da una confusión entre la derivada en un punto $f^{\prime}(a)$ y la función derivada $f^{\prime}(x)$. Posteriormente, se retomó este análisis desde el punto de vista de la teoría APOE en Badillo, Azcárate, y Font (2011).

Contreras, Luque, y Ordóñez (2003) llevan a cabo un estudio histórico-epistemológico en el que clasifican varias concepciones de la derivada y señalan los obstáculos resultantes de cada una de ellas. Concluyen que se produce una algebrización del concepto de derivada y no se analiza con suficiente profundidad su concepción como razón de cambio y como pendiente de la recta tangente.

La investigación sobre la derivada y sus problemas de aprendizaje no se ha ceñido al análisis de los productos del alumnado. Recientemente, Herrera, Velasco, y Ruiz-Fidalgo (2017) realizan un análisis de cómo dos manuales clásicos de cálculo presentan la derivada, siguiendo el marco teórico de Rico (1997) y el metodológico propuesto en Ibáñez y Llombart (2001).

Pino-Fan, Guzmán, Font, y Duval (2017) analizan el problema de la derivada de la función valor absoluto bajo la perspectiva comparada de la teoría de los registros de representación semióticos y del enfoque ontosemiótico de la instrucción matemática. Por su parte, Font, Trigueros, Badillo, y Rubio (2016) realizan un análisis paralelo sobre la descomposición genética de la derivada desde la teoría APOE y desde el enfoque ontosemiótico.

\section{Metodología}

\section{Objetivo}

La investigación que aquí se presenta pretende averiguar qué errores y dificultades encuentra el alumnado de $1^{\circ}$ de Bachillerato en el estudio de la derivada.

Este estudio trata de identificar y clasificar los errores más comunes y proponer pautas y procedimientos para su corrección $\mathrm{y}$, si es posible, eliminación. Al tratarse de un estudio exploratorio, la hipótesis de investigación como tal no debe ser formulada, aunque se sospecha que, como en estudios similares, la mayor parte de los errores serán causados por un desconocimiento de conceptos y habilidades necesarias para el estudio de la derivada: concepto de límite de una función y operaciones con funciones y con expresiones algebraicas.

\section{Población y muestra}

La población la constituye el alumnado de $1^{\mathrm{o}}$ de Bachillerato científico. La muestra seleccionada está formada por 27 estudiantes de $1^{\mathrm{o}}$ de Bachillerato de un instituto urbano de Oviedo (España), seleccionados por muestreo intencional no aleatorio, determinado por la disponibilidad de horas suficientes para acometer el estudio.

\section{Variables de estudio}

El análisis de los errores se ha organizado según los ámbitos de investigación propuestos por Sánchez-Matamoros et al. (2008) para la derivada:

a) Derivada de una función en un punto.

b) Relación entre la razón de cambio y la derivada.

c) Relación entre la derivada en un punto y la función derivada.

d) Sistemas de representación para el estudio de la derivada: interpretación geométrica de la derivada y estudio de la derivabilidad.

e) Regla de la cadena: derivación de funciones compuestas.

Las variables de estudio son los errores cometidos por el alumnado en ejercicios de este tipo.

\section{Recogida y análisis de datos}

Los datos fueron recogidos durante 15 sesiones didácticas a través de los siguientes medios:

- Hoja de registro de los errores observados durante la explicación de los contenidos en cada sesión, de acuerdo con la clasificación de Sánchez-Matamoros et al. (2008) (Anexo 1). Se registraron los errores que los estudiantes cometían, tanto en la resolución de los problemas y ejercicios propuestos, como en la comprensión de los conceptos y contenidos explicados. El profesor recogió, de modo sistemático, todos los errores cometidos por los estudiantes.

- Respuestas a los ejercicios complementarios realizados durante las sesiones (Anexo 2).

- Respuestas a los ejercicios de la prueba final (Anexo 3).

Previamente a la realización del estudio, se informó a todos los participantes del desarrollo de la investigación y de la finalidad de esta. Los 27 estudiantes o, en caso de menores de edad, sus representantes legales, dieron su consentimiento.

\section{Resultados}

En este apartado se describen los errores observados durante la explicación de los contenidos en cada sesión, en los ejercicios complementarios, y en los ejercicios de la prueba final, según la agrupación temática de la hoja de registro (véase Anexo 1).

\section{Errores observados en el desarrollo de las sesiones}

Derivada de una función en un punto y relación entre tasa de cambio y derivada

Los estudiantes ya habían manejado en otras asignaturas el concepto de tasa de cambio de una función en un intervalo y su aplicación física más elemental. Sin embargo, desconocían el concepto de derivada, su definición como límite de un cociente 
incremental y su relación con la tasa de cambio de una función. En el tratamiento en el aula del concepto y sus procedimientos de cálculo se observó lo siguiente:

- Algunos estudiantes se encontraban confusos al calcular la derivada de una función $f$ en el punto $x=a$, puesto que no identificaban el punto en cuestión como $(a, f(a))$. Sin embargo, cuando se les propuso calcular el punto de una función conocida su expresión algebraica y el valor de la abscisa, calcularon sin problemas el valor de la ordenada del punto. Por ello, se trata de un error por el lenguaje.
- A la hora del cálculo, cometieron algunos errores con el manejo del lenguaje algebraico (cambios de signos, simplificaciones mal resueltas, etc.). Más importantes y habituales fueron los fallos en el cálculo de límites (Figura 1). Esto refleja que algunos de los estudiantes no tienen interiorizado el concepto de límite, ya que confunden el límite de una función en un punto con el valor de la función en el punto. Son errores por aprendizaje deficiente de hechos, destrezas y conceptos previos.

Figura 1. Ejemplos de error en el cálculo de límites.

Ejemplo 1. $\lim _{h \rightarrow 0}(2+h)=2+h$. El resultado es $2+\mathrm{h}$.

Ejemplo 2. El límite $\lim _{h \rightarrow 0} 0 / h$ es una indeterminación $0 / 0$ y no tiene solución.

Función derivada

En este aspecto se produjeron frecuentes errores en operaciones con radicales, con fracciones algebraicas o con el cálculo de límites, debidos a un aprendizaje defectuoso de destrezas y conceptos previos.

Técnicas de derivación: derivada de funciones elementales y álgebra de derivadas

Se encontraron:
- Dificultades para asimilar las técnicas de derivación de las funciones logarítmicas, debido al desconocimiento del concepto de logaritmo y de sus propiedades.

- Confusiones entre la derivada del producto y del cociente con la derivada de la suma, aplicando pseudo-reglas como: "la derivada de un producto es el producto de las derivadas y la derivada de un cociente es el cociente de las derivadas" (Figura 2).

Figura 2. Ejemplos de aplicación de pseudo-reglas.

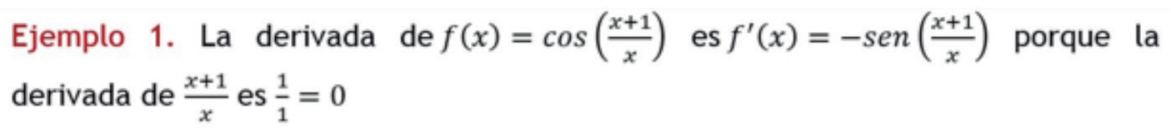

Ejemplo 2. La derivada de $f(x)=81 \cdot \ln ^{4}\left(x^{2}+3\right)$ es $f^{\prime}(x)=0$ porque la derivada de 81 es 0 .

Técnicas de derivación: la regla de la cadena

A este respecto algunos estudiantes mostraron:

- Dificultades a la hora de descomponer una función compuesta, identificando incorrectamente las componentes.

- Errores y desconocimiento de las reglas de derivación de las funciones elementales y de la propia regla de la cadena.

- Confusión entre el logaritmo de una potencia y la potencia de un logaritmo: muchos estudiantes consideraban erróneamente que $\ln ^{4}(x)=\ln \left(x^{4}\right)$, lo que los llevaba a aplicar la regla de la cadena de forma incorrecta.

Interpretación geométrica de la derivada

Algunos estudiantes mostraron:

- Dificultades a la hora de recordar la ecuación punto-pendiente y la ecuación explícita de una recta. Confusión a la hora de identificar la pendiente de una recta dada su ecuación con la ordenada en el origen, y viceversa. Fallos a la hora de determinar la ecuación de una recta conocidos un punto y su pendiente. Estos errores pueden ser causados por un aprendizaje defectuoso de la geometría analítica en el plano.
- Al calcular la recta tangente a una función en el punto $x=a$, dificultades para determinar la ordenada del punto y para identificar el parámetro $a$ como la abscisa del punto, y la $x$ como la variable independiente que toma el valor $a$. Esto se debe a dificultades en el lenguaje, específicamente a complejidades semióticas, ya que los mismos significantes adoptan significados diferentes en función del contexto.

Derivabilidad de funciones

Parte del alumnado mostró:

- Desconocimiento del cálculo de límites de funciones definidas a trozos debido a fallos en el cálculo de límites laterales.

- Confusión entre límite de una función en un punto y valor de la función en el punto, lo que conlleva errores en el cálculo de límites y en las condiciones de derivabilidad de una función en un punto. La Figura 3 muestra un ejemplo en el que, para estudiar la derivabilidad de una función, se omite el estudio de los límites laterales.

Figura 3. Ejemplo de error en el estudio de la derivabilidad.

$$
f(x)= \begin{cases}\frac{x}{1+e^{\frac{1}{x}}} & \text { si } x \neq 0 \\ 0 & \text { si } x=0\end{cases}
$$

La función es derivable en $x=0$ porque $\lim _{x \rightarrow 0} f^{\prime}(x)=f^{\prime}(0)=0$ 


\section{Errores observados en los ejercicios complementarios}

Ítem 1

En el primer apartado de este ítem los estudiantes tuvieron muchos problemas para llegar a la solución correcta, hubo muchas respuestas posiblemente debidas al azar, sin ninguna justificación y empleando estrategias incorrectas, etc. Esto contrasta con el segundo apartado, que fue resuelto correctamente por la mayoría de los estudiantes.

Debido a que el concepto clave en ambos apartados es el mismo (la derivada de una función en un punto) la forma de proporcionar la información sobre la función tuvo gran incidencia sobre la forma en que los estudiantes llegan a la solución correcta, a favor de la expresión funcional frente a la tabla.

Ítem 2

En el primer apartado de este ítem ocurrió algo similar al ítem 1. Los estudiantes encontraron dificultades para estudiar la derivada en un contexto distinto al analítico. La representación algebraica de las funciones domina la forma de proceder del alumnado, y se produce bloqueo si deben analizar funciones definidas por una tabla de valores o mediante su representación gráfica.

Una parte importante del alumnado no resolvió correctamente el segundo apartado. Algunos de los estudiantes eran incapaces de coordinar los contextos gráfico y analítico de la derivada, de forma que los consideraban como estrategias independientes para resolver diferentes problemas. Esto se combinó en otros casos con una interpretación incorrecta de la pendiente de la recta tangente y el signo de la derivada. Otros estudiantes no asociaron correctamente la pendiente de una recta con su crecimiento o decrecimiento.
La categorización de estos errores es múltiple. Por un lado, se produjeron errores debidos a asociaciones incorrectas entre la derivada en el contexto analítico y su interpretación geométrica. Por otro, a dificultades de lenguaje, al recibir la información en forma gráfica y, finalmente, al aprendizaje deficiente de conceptos, destrezas y habilidades básicas de geometría analítica.

\section{Errores observados en la prueba final}

Los resultados de los ítems 1 y 2 se muestran en la Tabla 1.

Tabla 1. Resultados de los ítems 1 y 2.

\begin{tabular}{lcc}
\hline Ítem & $\begin{array}{c}\text { Respuestas } \\
\text { correctas }\end{array}$ & $\begin{array}{c}\text { Respuestas } \\
\text { incorrectas }\end{array}$ \\
Ítem 1 & 19 & 8 \\
Ítem 2 - apartado 1 & 18 & 9 \\
Ítem 2 - apartado 2 & 20 & 7
\end{tabular}

Se registra un mayor número de respuestas correctas en ambos ítems. Además, podemos añadir que:

- Muchos errores en el ítem 1 se deben tanto a un desconocimiento de la definición de derivada de una función en un punto como a su aplicación a un caso concreto. Analizando las producciones auxiliares del alumnado para resolver el ejercicio, observamos dificultades a la hora de comprender la notación del límite. Por ejemplo, dos estudiantes escriben correctamente la definición de derivada en un punto (en términos de $h$ ), pero señalan una opción incorrecta. También se dieron errores debidos a dificultades del lenguaje en la notación de funciones (Figura 4).

Figura 4. Ejemplo de error debido a un fallo en la comprensión de la notación funcional.

$$
\begin{aligned}
& f(x)=\operatorname{sen} x \text { en el puito } x=\frac{\pi}{2} \\
& f(x)=\operatorname{sen} x\left(\frac{\pi}{2}\right) \Rightarrow f^{\prime}(x)=\cos x \cdot \frac{\pi}{2}=\frac{\cos x \cdot \pi}{2} \\
& f^{\prime}(x) \Rightarrow \lim _{x \rightarrow\left(\frac{\pi}{2}\right)}=\frac{\cos x}{x-\frac{\pi}{2}}
\end{aligned}
$$

- En el ítem 2 se observan respuestas incorrectas asociadas a errores causados por el desconocimiento de la función derivada o de las condiciones de derivabilidad, y por fa- llos en las técnicas de derivación y en las operaciones con números enteros (véase Figura 5).

Figura 5. Ejemplo de error en el concepto de función derivada y en la evaluación de funciones en puntos.

$$
\begin{aligned}
& f(x)=\left\{\begin{array}{l}
\frac{x^{2}}{2}+1 \text { si } x<2 \\
e^{2-x}+2 \text { si } x \geqslant 2
\end{array} \text { de } f \text { en } x=2 .\right. \\
& f^{\prime}\left(2^{-}\right)=\frac{x^{2}}{2}+1 \Rightarrow \frac{-2^{2}}{2}+1=-2+1=-1 \\
& f^{\prime}\left(2^{+}\right)=e^{2-x}+2 \Rightarrow e^{2-x}=e^{2-2}=e^{0}=1 .
\end{aligned}
$$


- En el apartado a del ítem 3 (composición de dos funciones), 11 estudiantes ni siquiera supieron descomponer la función. Este error se debe a un aprendizaje defectuoso del concepto de función compuesta. Sin embargo, 15 de los 16 que descompusieron la función correctamente, supieron aplicar la regla de la cadena y llegar a la solución adecuada.

- En el apartado b del ítem 3, solamente 6 estudiantes abordaron correctamente el ejercicio, y 5 de ellos llegaron a la solución correcta. El estudiante restante aplicó erróneamente la derivada del producto. Cabe destacar la gran cantidad de estudiantes que derivaron esta función mediante la regla de la cadena como si se tratase de una función potencial (Figura 6). La causa de este error es doble: aprendizaje defectuoso de las características de la función potencial, y uso de estrategias irrelevantes, ya que se aplican reglas apropiadas para las funciones potenciales en contextos inadecuados.

Figura 6. Ejemplos de error al derivar una función potencial/exponencial debido a la elección de estrategias irrelevantes.

\section{Ejemplo 1.}

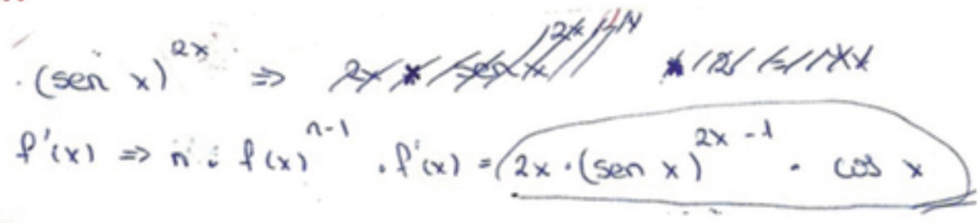

Ejemplo 2.

$$
\begin{aligned}
& f(x)=(\operatorname{sen} x)^{2 x} \\
& f^{\prime}(x)=2 x(\operatorname{sen} x)^{2 x-1} \cdot \cos x
\end{aligned}
$$

- En el apartado c del ítem 3 (composición de tres funciones), de nuevo la determinación de las funciones que componen la función produce la mayor parte de los fallos (16 de 27 estudiantes). La mayoría aplica correctamente la regla de la cadena y llega a la solución. En este caso, también el error es causado por un aprendizaje deficiente del concepto de función compuesta y de sus propiedades.

- En el apartado d del ítem 3, solamente un estudiante intentó simplificar la función aplicando las propiedades de los logaritmos, pero lo hizo de forma errónea aplicando una pseudo-regla: "el logaritmo de un producto es igual al producto de los logaritmos" (Figura 7). El error está causado por un desconocimiento de hechos y destrezas previos. El hecho de que ningún otro estudiante lo haya intentado puede deberse a que el concepto de logaritmo suele estudiarse en el contexto de los números reales, y no se traslada adecuadamente al contexto de las funciones. Al contrario que en los apartados a y c, en el d casi la totalidad de los estudiantes aplicaron erróneamente la regla de la cadena (la dificultad del ejercicio aumenta notablemente si no se aplican las propiedades de los logaritmos para simplificar la función). Otros errores fueron causados por aplicar la regla de la derivada del producto de forma incorrecta.

Figura 7. Ejemplo de error debido al desconocimiento de las propiedades de los logaritmos.

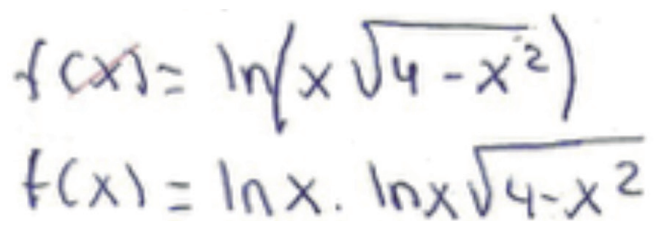

- En general, los estudiantes tienen grandes dificultades a la hora de simplificar las expresiones (Figura 8, ejemplo 1). Esto refleja en muchos casos un aprendizaje deficiente del manejo del lenguaje algebraico y, especialmente, de las propiedades de las funciones trigonométricas, siendo muy frecuentes errores (Figura 8, ejemplo 2).

Figura 8. Ejemplo de error a la hora de simplificar una expresión algebraica y desconocimiento de las funciones trigonométricas.

Ejemplo 1.

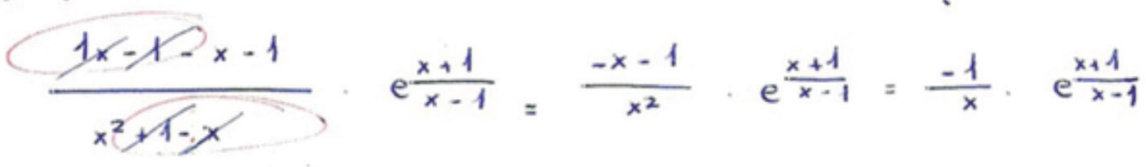

Ejemplo 2.

$$
f(x)=\cos ^{2}\left(\frac{x^{2}}{2}\right)=2 \cdot \cos \left(\frac{x^{2}}{2}\right)
$$


- Poco más de la mitad de los estudiantes (14 de 27) no resolvieron correctamente la parte del ítem 4 concerniente a la interpretación correcta del concepto de recta tangente, debido al desconocimiento del concepto de función y de sus propiedades. Los 13 restantes calculan correctamente el valor del parámetro $d$ y llegan a la relación $a+b+c=0$. De estos, 9 no aplican adecuadamente la interpretación geométrica de la derivada, por desconocimiento o por errores en el cálculo de la pendiente. Los otros 4 evalúan correctamente el valor de la derivada de la función en el punto. Además, 2 calculan bien el resto de los paráme- tros, mientras que 2 fallan en la resolución del sistema de ecuaciones lineales. También hubo estudiantes que intentaron resolver el problema sin el empleo de la derivada. En la Figura 9, se observa cómo el estudiante interpreta erróneamente el valor de la función en cada uno los puntos por el desconocimiento del concepto de función y de las coordenadas cartesianas de un punto en el plano. También se aprecia el empleo de estrategias irrelevantes combinadas con un aprendizaje deficiente de la resolución de sistemas de ecuaciones lineales.

Figura 9. Ejemplo de error a la hora de interpretar el valor de la función en cada uno los puntos por el desconocimiento del concepto de función $y$ de las coordenadas cartesianas de un punto en el plano.

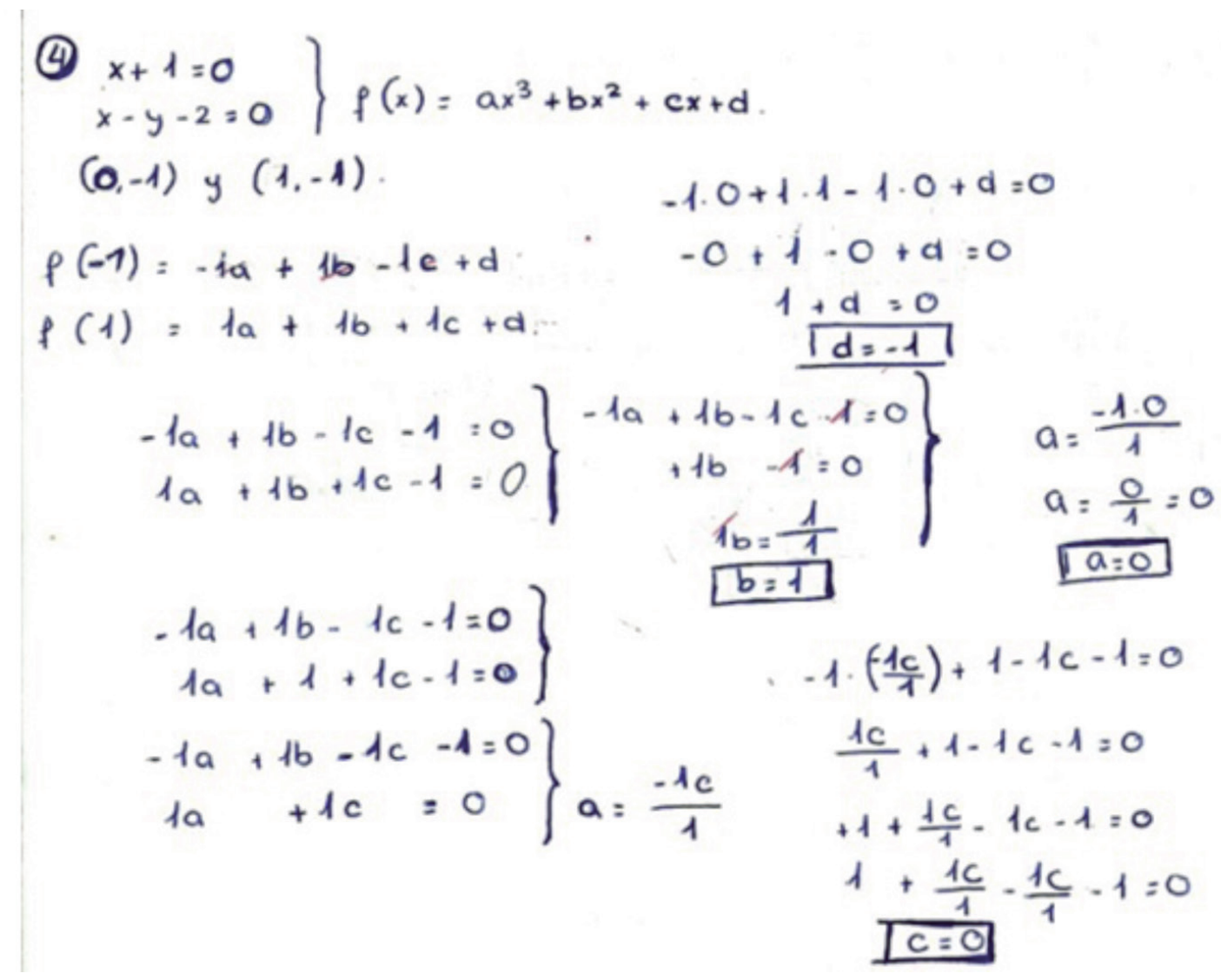

Los errores por estrategias irrelevantes son muy habituales en la resolución de este ítem. Por ejemplo, en la Figura 10 se pone de manifiesto que el estudiante, por encima de otros errores (falla al calcular el valor de la función $x=0$, desconoce el concepto de indeterminación), no tiene una estrategia clara para resolver el problema y se limita a manipular la expresión algebraica de la función y a calcular el punto de corte de las rectas dadas, que es un dato completamente irrelevante para resolver el problema. Se aprecia que tiene muchas dificultades para cambiar su estrategia inicial de resolución si esta no lleva a una solución. 
Figura 10. Ejemplo de errores en la resolución del ítem 4.

$$
\begin{aligned}
& f(x)=a x^{3}+\operatorname{lan}^{2}+c x+d \\
& y+1=0 \\
& y=-1 \\
& x-y-2=0 \\
& x+1-2=0 \\
& x-1=0 \\
& x=1
\end{aligned}
$$$$
\text { puntas: }(0,-1)(1,-1)
$$

$$
\begin{aligned}
f(t)= & a \cdot 1^{3}+b \cdot i^{2}+c \cdot 1+d=a \cdot d+b \cdot 1+c+d= \\
& a+b+c+d
\end{aligned}
$$

$$
\begin{aligned}
f(-1)= & a(-1)^{3}+b\left(-1^{2}\right)+c(-1)+d=a(-4)+b \cdot d+ \\
& -c+d=-a+b \cdot c+d \quad \text { Negativa } \\
f(0)= & a \cdot 0^{3}+b \cdot 0^{2}+c \cdot 0+d=a \cdot 0+b \cdot 0+0+d \\
& 0+0+0+d \rightarrow 0+d=1 \cdot 0 \\
& d=0
\end{aligned}
$$

$$
\begin{aligned}
& a x^{3}=-b x^{2}-c x-d \\
& a \cdot 1^{3}=-b \cdot 1^{2}-c \cdot 1-0 \\
& a \cdot 1=-b \cdot 1-c \\
& a=-b-c
\end{aligned}
$$

$$
\begin{aligned}
& a(-1)^{3}=-b(-1)^{2}-c(-1)-0 \\
& a(-1)=-b \cdot 1+c \\
& a(-1)=-b+c \\
& -a=-b+c \\
& a=b-c
\end{aligned}
$$

$$
\begin{aligned}
a\left(0^{3}\right) & =-b \cdot 0^{2}-c \cdot 0-0 \\
a \cdot 0 & =-b \cdot 0-0-0 \\
0 & =0-0-0
\end{aligned}
$$

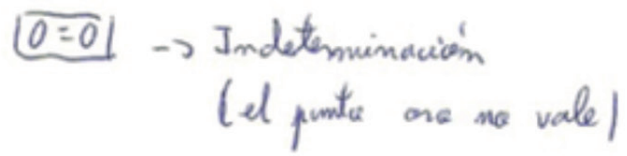

También se observa que una amplia mayoría de los estudiantes (23 de 27) no determinaron correctamente el valor de la derivada de la función en los puntos de tangencia, esto fue debido a dos errores principales, ambos causados por aprendizajes deficientes de hechos y destrezas: fallos en la determinación de la pendiente de una recta dada su ecuación, y desconocimiento

de la interpretación geométrica de la derivada. Por ejemplo, en la Figura 11 el estudiante calcula el punto de corte de las rectas, y además asocia el valor de la abscisa del punto con el valor de la pendiente de la recta, lo que refleja un aprendizaje defectuoso del concepto de pendiente de una recta.

Figura 11. Ejemplo de error debido a un fallo en el cálculo de la pendiente de la recta.

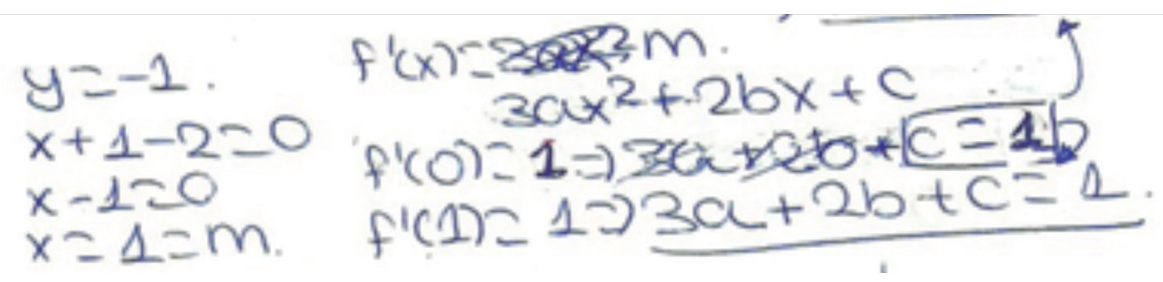

La Figura 12 resume el porcentaje de errores encontrados en cada ítem de la prueba final y las causas a las que se asocian.
Como vemos, la mayoría de los errores son debidos a aprendizajes deficientes de hechos, destrezas y conceptos previos. 
Figura 12. Gráfico resumen de los errores encontrados.

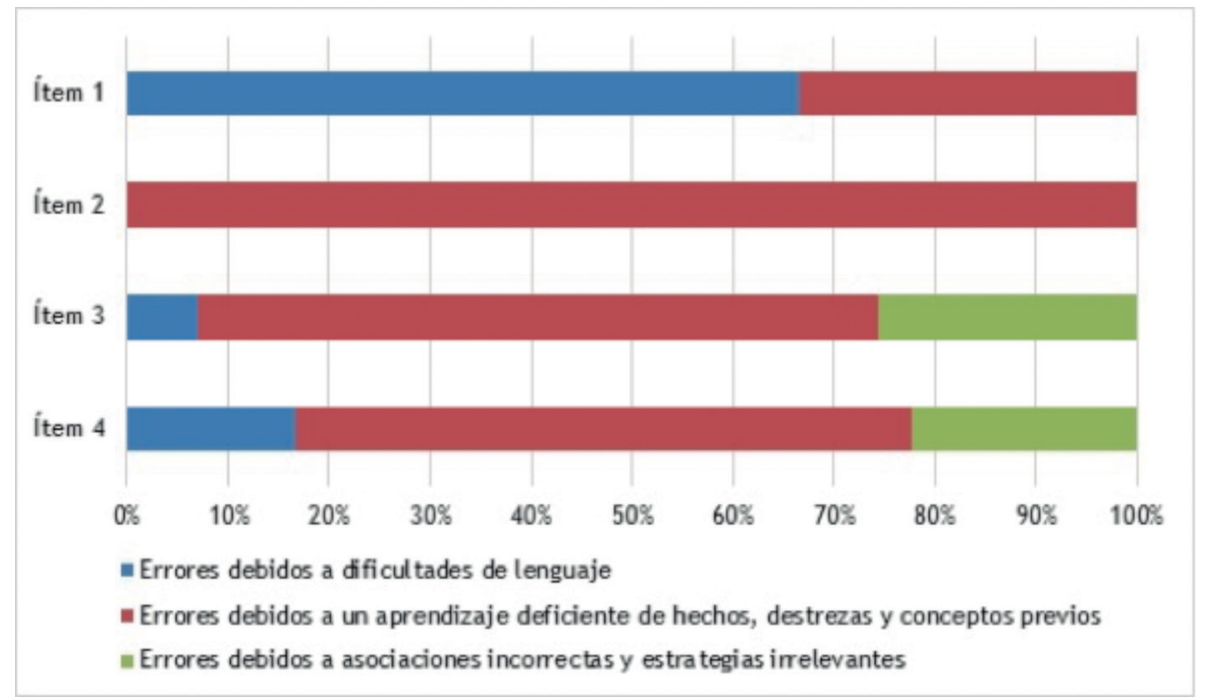

Los resultados obtenidos corroboran que la mayoría de los errores de los estudiantes se debe a un aprendizaje defectuoso de conceptos y habilidades previas. Además, reflejan errores en geometría analítica básica relacionados con el concepto de recta tangente y pendiente de una recta.

\section{Discusión y conclusiones}

El trabajo aporta un enfoque novedoso respecto a los trabajos anteriores sobre errores, al realizar el seguimiento de un grupo de estudiantes durante 15 sesiones docentes, además de varias pruebas de evaluación, en lugar de evaluar los errores de modo puntual en una prueba o en una sesión. Ello da mayor consistencia a los resultados y respalda con más firmeza las consideraciones que realizaremos a continuación.

A modo de recapitulación, y agrupando lo observado en todos los aspectos evaluados, concluimos que, a pesar de encontrar una asimilación adecuada de procedimientos sistemáticos (por ejemplo, el cálculo de derivadas mediante su definición como límite), se constatan los siguientes errores y dificultades en el aprendizaje de la derivada:

- Errores a la hora de operar y simplificar expresiones algebraicas. Por ejemplo, operar con un signo negativo como si fuera positivo.

- Dificultad a la hora de cambiar las estrategias escogidas para la resolución de problemas si estas no son adecuadas. Por ejemplo, perseverancia en aplicar la regla de la cadena sin intentar simplificar la expresión utilizando logaritmos.

- Incapacidad para analizar funciones dadas en forma de tabla o mediante su representación gráfica.

- Incapacidad para resolver aspectos relacionados con la interpretación geométrica de la derivada. Especialmente, la confusión entre el valor de la función y el valor de la pendiente en un punto.

- Ausencia de la comprobación de la coherencia de los resultados obtenidos.

Este estudio corrobora que los estudiantes cometen menos errores a la hora de realizar procedimientos sistemáticos, pero fallan con mucha asiduidad en su interpretación y comprensión
(Mallart, 2014). Los resultados anteriores evidencian la vinculación entre estos errores y un manejo incorrecto del lenguaje matemático. También se ha constatado la dificultad del manejo de la regla de la cadena, y de algunas simplificaciones con funciones logarítmicas o exponenciales que facilitan su aplicación, coincidiendo con los resultados de investigaciones anteriores (Clark et al., 1997).

En este trabajo se concluye que los estudiantes consideran el aspecto analítico y geométrico de la derivada como problemas independientes que se resuelven con estrategias diferentes, algo que ya apuntó Asiala et al. (1997). Además, se refleja que los estudiantes adquieren con menos dificultades el concepto de derivada en el ámbito algebraico que en el geométrico, produciéndose una algebrización del concepto de derivada, que resulta en una mayor facilidad para manejarse con el lenguaje funcional respecto al geométrico (Contreras et al., 2003; Habre y Abboud, 2004).

También coincidimos con Nortes Checa y Nortes Martínez-Artero (2010) en que algunos errores al manejar expresiones algebraicas impiden llegar a la solución correcta, a pesar de que las técnicas de derivación estén bien empleadas. Asimismo, los estudiantes tienen dificultades a la hora de cambiar sus estrategias a pesar de que sean infructuosas para resolver el problema, y muchos de los errores cometidos son consecuencia de la ausencia de un análisis coherente de los resultados por parte de los estudiantes.

De acuerdo con los resultados obtenidos en este estudio y a las conclusiones expuestas, consideramos que es importante tener en cuenta los siguientes apartados cuando se aborden en el aula de Bachillerato la enseñanza y el aprendizaje de la derivada.

En primer lugar, debemos asegurarnos de que los estudiantes manejan sin grandes dificultades el concepto de límite de una función en un punto, así como el manejo de indeterminaciones. El desconocimiento de las funciones elementales y sus características principales también son fuente de bastantes errores, así como la falta de asimilación de los conceptos básicos de la geometría analítica (pendiente de una recta e interpretación de su signo, crecimiento y decrecimiento, ecuación punto-pendiente, etc.). Lo anterior dificulta, en algunos casos, el paso a la Educación Superior (Huidobro, Méndez, y Serrano, 2010; Rodríguez-Muñiz, Díaz, Mier, y Alonso, 2016).

Un segundo aspecto es el relacionado con las funciones. Como se señaló en Contreras et al. (2003), y así lo hemos corro- 
borado con nuestro estudio, la idea predominante que los estudiantes tienen sobre la función es la analítica, lo que hace que aparezcan muchas dificultades cuando se estudian gráficas o funciones dadas en forma de tabla. Por ello, se debe fomentar más ese tratamiento de la función, con el objeto de que conceptos y propiedades muy relacionados con la geometría o la variación, como es el caso de la derivada, sean abordados desde un punto de vista global, tal y como señalaba Zandieth (2000) con su metáfora del puzle, que integre todos los significados de la derivada, y dando un peso mayor al análisis visual (Badillo, Figueiras, Font, y Martínez, 2013), al estudio de la variación (García González y Dolores Flores, 2016) y al análisis de las conexiones entre las distintas representaciones (García-García y Dolores-Flores, 2017). Por otro lado, hemos constatado la dificultad del manejo de las tablas de valores. Font (2000) defendía que su uso facilitaba la comprensión de la idea de tasa de variación. Nuestros resultados muestran que, por el contrario, esto genera numerosos errores, quizá precisamente por la poca familiaridad en su uso.

Las dificultades del lenguaje también suponen un escollo importante para muchos estudiantes (Oliveira, Mazer, Guillaumon, y Fernández, 2014). Los conflictos semióticos han sido tratados en varios de los trabajos ya citados anteriormente y se han puesto en evidencia en los errores estudiados en este trabajo. La incidencia de estos problemas llega incluso a los libros de texto (Badillo, Font, y Azcárate, 2005; Díaz-Levicoy, Giacomone y Arteaga, 2017). Por consiguiente, es necesario manejar un lenguaje matemático no equívoco, lo cual requiere un estudio profundo de los materiales utilizados en el aula.

Se debe fomentar en el aula el análisis lógico y coherente de los conceptos matemáticos $\mathrm{y}$, especialmente, de las soluciones, para corroborar su coherencia con el ejercicio o problema que se resuelve (Nortes Checa y Nortes Martínez-Artero, 2010). Asimismo, entendemos que un trabajo de este tipo puede servir como base para desarrollar la habilidad de anticipación en el profesorado de matemáticas de Secundaria en formación, en la línea de Sánchez-Matamoros, Fernández, y Llinares (2015) y Fernández, Sánchez-Matamoros, Moreno, y Callejo (2018)

Para finalizar, apuntamos las principales limitaciones del presente trabajo. Consideramos que el tamaño de la muestra es adecuado, si bien haber podido comparar los resultados del grupo estudiado con los de un grupo instruido por otro profesor habría permitido aislar el posible efecto del docente en los resultados. El hecho de que el mismo profesor impartiese la docencia $\mathrm{y}$, a la vez, registrase los errores cometidos, afectó a la capacidad para recoger datos durante las sesiones, aunque no afectó al registro de los errores procedentes de los ejercicios y exámenes.

Metodológicamente observamos que la clasificación de Radatz confiere mucho peso a los errores por aprendizaje deficiente de conceptos y procedimientos previos (como ya apuntaba Rodríguez-Muñiz y Candás, 2017). Ello no ha de significar necesariamente que no ocurra así, pero a nuestro juicio distorsiona el conocimiento obtenido sobre el tema en cuestión. En otras palabras, encontrar como causa mayoritaria de los errores que no se haya aprendido correctamente lo previo o bien pone la lupa sobre la propia clasificación utilizada o bien revela una situación que conlleva a cuestionar seriamente tanto el contenido como la estructura del currículo impartido. Este refinamiento de la clasificación, así como el estudio del currículo en el caso español, son dos de los problemas que se abren a partir de este trabajo

\section{Referencias bibliográficas}

Artigue, M. (1995). La enseñanza de los principios del cálculo: Problemas epistemológicos, cognitivos y didácticos. En M.
Artigue, R. Douady, L. Moreno y P. Gómez (Eds.), Ingeniería didáctica en educación matemática (pp. 97-140). México: Grupo Editorial Iberoamérica.

Asiala, M., Cottrill, J., Dubinsky, E., y Schwingendorf, K. (1997). The development of student's graphical understanding of the derivate. Journal of Mathematical Behavior, 16(4), 399-431.

Azcárate, C. (1990). La velocidad: Introducción al concepto de derivada. Tesis doctoral (no publicada). Universidad Autónoma de Barcelona. España.

Azcárate, C., Casadelvall, M., Casellas, E., y Bosch, E. (1996) Cálculo diferencial e integral. Madrid: Síntesis.

Badillo, E. (2003). La derivada como objeto matemático y como objeto de enseñanza y aprendizaje en profesores de matemáticas de Colombia. Tesis doctoral (no publicada). Universidad Autónoma de Barcelona. España.

Badillo, E., Font, V., y Azcárate, C. (2005). Conflictos semióticos relacionados con el uso de la notación incremental y diferencial en libros de física y de matemática del Bachillerato. Enseñanza de las Ciencias, Número Extra, 1-6.

Badillo, E., Azcárate, C., y Font, V. (2011). Análisis de los niveles de comprensión de los objetos $\mathrm{f}^{\prime}(\mathrm{a}) \mathrm{y} \mathrm{f}^{\prime}(\mathrm{x})$ en profesores de matemáticas. Enseñanza de las Ciencias, 29(2), 191-206.

Badillo, E., Figueiras, L., Font, V., y Martínez, M. (2013). Visualización gráfica y análisis comparativo de la práctica matemática en el aula. Enseñanza de las Ciencias, 31(3), 207-225.

Bocco, M., y Canter, C. (2010). Errores en geometría: Clasificación e incidencia en un curso preuniversitario. Revista Iberoamericana de Educación, 53(2), 1-13.

Cantoral, R. y Farfán, R. (2003). Mathematics education: A vision of its evolution. Educational Studies in Mathematics, 53(3), 255270.

Clark, J.M., Cordero, F., Cottrill, J., Czarnocha, B., DeVries, D.J., St. John, D., Tolias, G., y Vidakovic, D. (1997). Constructing a schema: The case of the chain rule. Journal of Mathematical Behavior, 14(4), 345-364.

Contreras, A., Luque, L., y Ordóñez, L. (2003). Una perspectiva de la enseñanza aprendizaje de la continuidad y la derivada de una función en Bachillerato y Universidad. Revista de Educación, 331, 399-419.

Díaz-Levicoy, D., Giacomone, B., y Arteaga, P. (2017). Caracterización de los gráficos estadísticos en libros de texto argentinos del segundo ciclo de Educación Primaria. Profesorado. Revista de currículum y formación del profesorado, 21(2), 299-325.

Engler, A., Gregorini, M.I., Müller, D., Vrancken, S., y Hecklein, M. (2004). Los errores en el aprendizaje de la matemática. Boletín de la SOAREM, 6, 23-32.

Fernández, C., Sánchez-Matamoros, G., Moreno, M., y Callejo, M.L. (2018). La coordinación de las aproximaciones en la comprensión del concepto de límite cuando los estudiantes para profesor anticipan respuestas de estudiantes. Enseñanza de las Ciencias, 36(1), 143-162.

Font, V. (2000). Procediments per obtenir expressions simbòliques a partir de gràfiques. Aplicacions a la derivada. Tesis doctoral (no publicada), Universidad de Barcelona. España.

Font, V., Trigueros, M., Badillo, E., y Rubio, N. (2016). Mathematical objects through the lens of two different theoretical perspectives: APOS and OSA. Educational Studies in Mathematics, 91, 107-122.

Franchi, L., y Hernández de Rincón, A.I. (2004). Tipología de errores en el área de la geometría plana. Educere, 8(24), 63-71.

García-García, J., y Dolores-Flores, C. (2018). Intra-mathematical connections made by high school students in performing Calculus tasks. International Journal of Mathematical Education in Science and Technology, 49(2), 227-252. 
García González, M.S., y Dolores Flores, C. (2016). Diseño de una situación de aprendizaje para la comprensión de la derivada. UNIÓN, 46, 49-70.

Habre, S., y Aboud, M. (2006). Student's conceptual understanding of a function and its derivative in an experimental calculus course. Journal of Mathematical Behavior, 25(1), 57-72.

Herrera, M., Velasco, M., y Ruiz-Hidalgo, J. (2017). Comparando textos de cálculo: El caso de la derivada. PNA, 11(4), 280-306.

Huidobro, J.A., Méndez, M.A., y Serrano, M.L. (2010). Del Bachillerato a la Universidad: las matemáticas en las carreras de ciencias y tecnología. Aula Abierta, 38(1), 71-80.

Ibáñez, I. y Llombart, J. (2001). La comparación de textos en historia de la ciencia: una propuesta metodológica. Llull, 24, 131148.

Mallart, A. (2014). La resolución de problemas en la prueba de Matemáticas de acceso a la universidad: Procesos y errores. Educatio Siglo XXI, 32(2), 233-254.

Martínez Jurado, L., y Francisco Solano, A.E. (2010). ¿Diferentes representaciones de la derivada? Las dificultades que tienen los estudiantes de nivel superior para relacionarlas. Recuperado de http://www.repositoriodigital.ipn.mx/handle/123456789/3274

Montiel, G. (2005). Interacciones en un escenario en línea. El papel de la socioepistemología en la resignificación del concepto de derivada. RELIME, 8(2), 219-235.

Movshovitz-Hadar, N., Zaslavsky, O., y Inbar, S. (1987). An empirical classification model for errors in high school mathematics. Journal for Research in Mathematics Education, 18(1), 3-14.

Nortes Checa, A., y Nortes Martínez-Artero, R. (2010). Resolución de problemas de matemáticas en las pruebas de acceso a la universidad. Errores significativos. Educatio Siglo XXI, 21(1), 317-342.

Oliveira, M., Mazer, S.M., Guillaumon, M.L., y Fernández, E. (2014). Análisis de la producción científica en Brasil sobre dificultades de aprendizaje una revisión bibliométrica. Aula Abierta, 42(1), 31-38.

Ortega, T. y Sierra M. (1998). El concepto de derivada: algunas indicaciones para su enseñanza. Revista Interuniversitaria de Formación del Profesorado 32, 87-115.

Orton, A. (1983). Student's understanding of differentiation. Educational Studies in Mathematics, 14(3), 235-250.
Pino-Fan, L., Guzmán, I., Font, V., y Duval, R. (2017). Analysis of the underlying cognitive activity in the resolution of a task on derivability of the absolute-value function: Two theoretical perspectives. PNA, 11(2), 97-124

Radatz, H. (1979). Error analysis in mathematics education. Journal for Research in Mathematics Education, 10(3), 162-172.

Radatz, H. (1980). Students' errors in the mathematical learning process: A survey. For the Learning of Mathematics, 1(1), 16-20.

Rico, L. (1997). Consideraciones sobre el currículo de matemáticas para educación secundaria. En L. Rico (Coord.), La educación matemática en la enseñanza secundaria (pp. 15-38). Barcelona, España: Horsori.

Rico, L. (1998). Educación Matemática. Errores y dificultades de los estudiantes. Resolución de problemas. Evaluación. Historia. En J. Kilpatrick, L. Rico, y P. Gómez (Eds.), Educación matemática (pp. 69-108). Bogotá: Universidad de los Andes.

Rodríguez-Muñiz, L.J., y Candás, P. (2017). Análisis de los errores cometidos al resolver un límite en exámenes de PAU. En FESPM (Ed.), Libro de Actas. VIII Congreso Iberoamericano de Educación Matemática, CB-361 (pp. 309-317). Madrid: FESPM.

Rodríguez-Muñiz, L.J., Díaz, P., Mier, V., y Alonso, P. (2016). Washback Effect of University Entrance exams in Applied Mathematics to Social Sciences. PLoS ONE, 11(12), e0167544.

Sánchez-Matamoros, G., García, M., y Llinares, S. (2008). La comprensión de la derivada como objeto de investigación en didáctica de la matemática. Revista Latinoamericana de Investigación en Matemática Educativa, 11(2), 267-296.

Sánchez-Matamoros, G., Fernández, C., y Llinares, S. (2015). Developing pre-service teachers' noticing of students' understanding of the derivative concept. International Journal of Science and Mathematics Education, 13, 1305-1329.

Socas, M. (1997). Dificultades, obstáculos y errores en el aprendizaje de las Matemáticas en la Educación Secundaria. En L. Rico (Coord.), La Educación Matemática en la Enseñanza Secundaria (pp. 125-154). Barcelona: Horsori.

Zandieth, M. (2000). A theoretical framework for analyzing student understanding of the concept of derivate. En E. Dubinsky, A. Shoenfeld, y J. Kaput (Eds.), Research in Collegiate Mathematics Education. IV CBMS Issues in Mathematics Education (volume 8, pp. 103-127). Providence, USA: American Mathematical Society. 
Anexo 1. Hoja de registro de los errores observados durante la explicación de los contenidos

\begin{tabular}{|c|c|}
\hline Apartado & Observaciones \\
\hline \multirow{3}{*}{$\begin{array}{l}\text { Derivada de una función } \\
\text { en un punto }\end{array}$} & Dificultades de lenguaje \\
\hline & $\begin{array}{l}\text { Aprendizaje deficiente de hechos, } \\
\text { destrezas y conceptos previos }\end{array}$ \\
\hline & Otros \\
\hline \multirow[t]{4}{*}{ Función derivada } & Dificultades de lenguaje \\
\hline & $\begin{array}{l}\text { Aprendizaje deficiente de hechos, } \\
\text { destrezas y conceptos previos }\end{array}$ \\
\hline & $\begin{array}{l}\text { Asociaciones incorrectas o estrategias } \\
\text { irrelevantes }\end{array}$ \\
\hline & Otros \\
\hline \multirow{4}{*}{$\begin{array}{l}\text { Técnicas de derivación } \\
\text { elementales }\end{array}$} & Desconocimiento \\
\hline & $\begin{array}{l}\text { Aprendizaje deficiente de hechos, } \\
\text { destrezas y conceptos previos }\end{array}$ \\
\hline & $\begin{array}{l}\text { Asociaciones incorrectas o estrategias } \\
\text { irrelevantes }\end{array}$ \\
\hline & Otros \\
\hline \multirow{5}{*}{$\begin{array}{l}\text { Técnicas de derivación II. } \\
\text { La regla de la cadena }\end{array}$} & Dificultades de lenguaje \\
\hline & Desconocimiento \\
\hline & $\begin{array}{l}\text { Aprendizaje deficiente de hechos, } \\
\text { destrezas y conceptos previos }\end{array}$ \\
\hline & $\begin{array}{l}\text { Asociaciones incorrectas o estrategias } \\
\text { irrelevantes }\end{array}$ \\
\hline & Otros \\
\hline \multirow{6}{*}{$\begin{array}{l}\text { Interpretación geométrica } \\
\text { de la derivada }\end{array}$} & Dificultades de lenguaje \\
\hline & Desconocimiento \\
\hline & $\begin{array}{l}\text { Aprendizaje deficiente de hechos, } \\
\text { destrezas y conceptos previos }\end{array}$ \\
\hline & $\begin{array}{l}\text { Asociaciones incorrectas o estrategias } \\
\text { irrelevantes }\end{array}$ \\
\hline & $\begin{array}{l}\text { Dificultades para obtener información } \\
\text { espacial }\end{array}$ \\
\hline & Otros \\
\hline \multirow{6}{*}{$\begin{array}{l}\text { Derivabilidad de } \\
\text { funciones }\end{array}$} & Dificultades de lenguaje \\
\hline & Desconocimiento \\
\hline & $\begin{array}{l}\text { Aprendizaje deficiente de hechos, } \\
\text { destrezas y conceptos previos }\end{array}$ \\
\hline & $\begin{array}{l}\text { Asociaciones incorrectas o estrategias } \\
\text { irrelevantes }\end{array}$ \\
\hline & $\begin{array}{l}\text { Dificultades para obtener información } \\
\text { espacial }\end{array}$ \\
\hline & Otros \\
\hline
\end{tabular}




\section{Ítem 1.}

1. Si de la función $f$ se conocen los datos que figuran en la siguiente tabla, ¿cuál sería el valor estimado de $f(1)$ ?

$\begin{array}{lcc}\text { a) } 1 & 0.8 & 3.34 \\ \text { b) } 2 & 0.9 & 3.81 \\ \text { c) } 3 & 0.95 & 3.9025 \\ \text { d) } 4 & 1 & 4 \\ & 1.01 & 4.0201 \\ & 1.1 & 4.21 \\ & 1.15 & 4.62\end{array}$

2. Halla, mediante la definición, la derivada de $f(x)=x^{2}-4$ en $x=1$.

3. Los siguientes límites son una derivada, ¿de qué función y en qué punto?
a) $\lim _{h \rightarrow 0} \frac{2 \cdot(5+h)^{3}-2 \cdot 5^{3}}{h}$
b) $\lim _{x \rightarrow 3} \frac{x^{3}+x-30}{x-3}$

\section{f́tem 2.}

1. La gráfica de más abajo corresponde a una función $f$ de la que se conocen además los valores que figuran en la tabla siguiente:

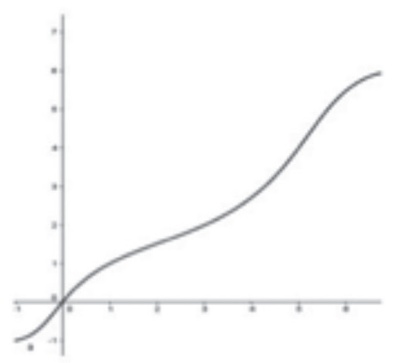

\begin{tabular}{cc}
$x$ & $f(x)$ \\
\hline 3 & 1.25 \\
5 & 3.25 \\
5.5 & 4.0625 \\
5.9 & 4.8025 \\
6 & 5
\end{tabular}

En consecuencia, de las afirmaciones que siguen, ¿cuál puede ser cierta?
a) $\lim _{x \rightarrow 6} f(x)=2$
b) $f^{\prime}\left(6^{-}\right)=2$
c) $f^{\prime}\left(3^{-}\right)=-1,25$
d) $\lim _{x \rightarrow 3} f(x)=-2,5$

2. La siguiente figura muestra las gráficas de dos funciones. Una de ellas es una función $f$ y la otra su derivada $f$. Asocia razonadamente cada función con su gráfica.

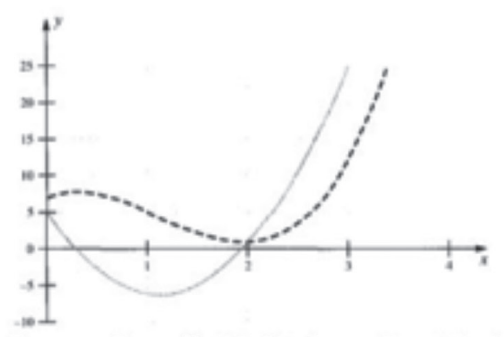

Fuente: Purcell, E.J., Varberg, D., y Rigdon, S.E. (2007). Cálculo. México: Pearson Educación. 
Ítem 1. ¿Cuál de las siguientes expresiones corresponde a la derivada de $f(x)=\operatorname{sen}(x)$ en el punto $x=\frac{\pi}{2}$ ?
a) $\lim _{h \rightarrow \frac{\pi}{2}} \frac{\operatorname{sen}\left(\frac{\pi}{2}+h\right)-1}{h}$
b) $\lim _{x \rightarrow \frac{\pi}{2}} \frac{\cos (x)}{x-\frac{\pi}{2}}$
c) $\lim _{x \rightarrow 0} \frac{\operatorname{sen}(x)-1}{x-\frac{\pi}{2}}$
d) $\lim _{x \rightarrow \frac{\pi}{2}} \frac{\operatorname{sen}(x)-1}{x-\frac{\pi}{2}}$

Item 2. Dada la función $f(x)=\left\{\begin{array}{ll}\frac{x^{2}}{2}+1 & \text { si } x<2 \\ e^{2-x}+2 & \text { si } x \geq 2\end{array}\right.$.

1. ¿Cuál es el valor de las derivadas laterales de $f$ en $x=2$ ?
a) $f^{\prime}\left(2^{-}\right)=f^{\prime}\left(2^{+}\right)=2$
b) $f^{\prime}\left(2^{-}\right)=f^{\prime}\left(2^{+}\right)=-1$
c) $f^{\prime}\left(2^{-}\right)=2$ y $f^{\prime}\left(2^{+}\right)=-1$
d) $f^{\prime}\left(2^{-}\right)=-1$ y $f^{\prime}\left(2^{+}\right)=2$

2. ¿Es derivable $f$ en $x=2$ ? En caso afirmativo, ¿cuál es el valor de su derivada en $x=2$ ?
a) No, al ser distintas las derivadas laterales de $f$ en $x=2$.
b) No, porque $f$ no es continua en $x=2$.
c) Sí, y su derivada en $x=2$ es -1 .
d) Si, y su derivada en $x=2$ es 2 .

Ítem 3. Halla la derivada de las siguientes funciones y simplifica todo lo posible el resultado:
a) $f(x)=e^{\frac{x+1}{x-1}}$
b) $f(x)=(\operatorname{sen}(x))^{2 x}$
c) $f(x)=\cos ^{2}\left(\frac{x^{2}}{2}\right)$
d) $f(x)=\ln \left(x \sqrt{4-x^{2}}\right)$

Ítem 4. Las rectas $y+1=0$ y $x-y-2=0$ son tangentes a la función $f(x)=a x^{3}+b x^{2}+c x+d$ en los puntos $(0,-1)$ y $(1,-1)$, respectivamente. Halla los valores de $a, b, c$ y $d$. 